\title{
Correlation of oncologic long-term results and genetic instability in soft tissue sarcomas
}

\author{
Reiner Wirbel ${ }^{1}$, Steffen Weber ${ }^{2}$, Joachim Hans ${ }^{2}$, Wolf Mutschler ${ }^{3}$ \\ ${ }^{1}$ Department of Trauma, hand, and Reconstructive Surgery, Verbundkrankenhaus Bernkastel, Wittlich, Wittlich, Germany \\ ${ }^{2}$ Department of Trauma, Hand, and reconstructive Surgery University Hospital Saarland, Homburg, Germany \\ ${ }^{3}$ Department of Surgery, Ludwig, Maximilian University, Munich, Germany
}

\section{Email address:}

reiner.wirbel@web.de (R. Wirbel)

\section{To cite this article:}

Reiner Wirbel, Steffen Weber, Joachim Hans, Wolf Mutschler. Correlation of Oncologic Long-Term Results and Genetic Instability in Soft Tissue Sarcomas. Cancer Research Journal. Vol. 2, No. 4, 2014, pp. 74-81. doi: 10.11648/j.crj.20140204.13

\begin{abstract}
Purpose: Soft tissue sarcomas (STS) represent a heterotop group of tumours. Microsatellite instabilities (MSI) and loss of heterozygosity ( $\mathrm{LOH}$ ) as phenomena of a genetic instability should be analysed in STS and correlated with the long-term oncologic outcome. Methods: Patients treated for a STS with a follow-up of at least 10 years were included. Thus, 86 patients (mean age 50.5 years, range 16-86 years) treated for a STS between 1993 and 2000 were routinely controlled every 6 months. Incidence of local recurrences, distant metastases, and overall survival were analysed. Sixty-six tumour samples were available for microsatellite analysis using the former traditional method of PCR amplification at 6 loci in the neighbourhood of hMSH2, hMLH1, p53, p16, rb1, and hTR. Results: There were 30 low-grade and 56 high-grade sarcomas. The mean follow-up was 144 months (120-192 months). Twenty-nine patients died of their disease. Local recurrences were seen in 13 patients, whereas metastases were noticed in 23 patients. The overall survival was dependent on the tumour stage $(p<0.05)$, whereas the local tumour control (incidence of local recurrence) was influenced by the surgical margin achieved $(\mathrm{p}<0.05)$. The molecular biologic findings revealed $67 \%$ of the investigated loci as informative. MSI was found in $6.8 \%$ of the informative loci, whereas $\mathrm{LOH}$ in $18.8 \%$, respectively. $\mathrm{LOH}$ was present in high-grade tumours in $23.8 \%$, whereas in $1.7 \%$ in low-grade tumours. In high-grade sarcomas, the 5 -year and 10 -year survival probabilities were significantly lower in LOH-positive tumours $(48.6 \%$ and $38 \%)$ than in LOH-negative tumours $(72.5 \%$ and $62 \%$ ). Conclusion: The overall survival in soft tissue sarcoma is mainly influenced by the tumour stage. In high-grade sarcomas, the survival rate will drop even after 5 years. The detection of loss of heterozygosity represents a negative prognostic predictor in high-grade sarcomas. Microsatellite instability is a rare phenomenon supposing no relevance in the oncogenesis and tumour progression of soft tissue sarcomas.
\end{abstract}

Keywords: Soft Tissue Sarcoma, Oncologic Result, Genetic Instability, Microsatellite Instability

\section{Introduction}

Soft tissue sarcomas (STS) are rare with an incidence of $20-25$ per million. There are more than 50 histologic subtypes of STS. Although there exists a line of differentiation in most STS, many of these tumours show heterogeneous morphology and multiple, often complex, cytogenic aberrations $[1,2]$.

There exist inconsistent molecular biological data concerning different types of STS [3 - 9].

Further classification and elucidation of the molecular pathogenesis of STS is highly desirable. Genetic instability is a paramount feature of cancer which leads to accumulation of genetic alterations that varies from subtle changes in DNA sequence to chromosomal abnormalities $[1,2,10]$.

Microsatellite instability (MSI) is a particular type of genetic instability affecting short sequences of DNA repeats found throughout the genome [10]. It is considered as the appearance of replication errors which present mutations close to the genes of the mismatch repair (MMR) family [5, $6,11-15]$. The relevance of MSI is well known in different cancers, especially in the hereditary nonpolyposis colorectal cancer (HNPCC) [16, 17].

The loss of heterozygosity ( $\mathrm{LOH}$ ) of diferent tumour suppressor genes is considered to be a further well- 
established marker of the genomic instability in different types of cancers $[1,2,16,18]$.

But there exist different statements in the literature about the relevance of MSI and LOH in sarcomas. Ewing's sarcoma, rhabdomyosarcoma, liposarcoma, and leiomyosarcoma are the most frequently examined sarcomas [4 9, 14, 15, 19 - 27]. Microsatellite analysis is mostly reported at the neighborhood of the tumour suppressor genes (p53, rb1, hTR, p16) and of the mismatch repair genes (hMLH1, hMSH2) [5, 6, 11 - 15]. Mostly due to small series the role of genetic instability in sarcomas remains unclear, especially concerning the clinical outcome and the biological behaviour [3, $7-9,21]$

Most of the clinical studies of patients with soft tissue sarcomas cannot be assessed concerning the oncologic long-term outcome due to the mostly limited follow-up's of five years. Studies with real long-term results of oncological outcome are rare.

After the promising results of studying genetic instability in the defined entity of intraosseous leiomyosarcoma [9], the aim of the present study was to evaluate the occurrence of genetic instability in different STS. The correlation with the oncologic long-term outcome was established by using the findings of patients with a follow-up of at least 10 years.

\section{Patients and Methods}

\subsection{Patients and Tumour Samples}

From January 1993 to December 2010, 203 cases of soft tissue sarcomas located at the trunk or the extremities were treated surgically. To get information about the long-term prognosis, patients with a minimum follow-up of 10 years were selected. Thus, patients treated between 1993 and 2000 were included in this study.

There were 86 patients (51 men, 35 women) with an average age of 50.5 years (range, 16-86 years). The different histologies of STS include malignant fibrous histiocytoma $(\mathrm{MFH})(\mathrm{n}=26)$, liposarcoma $(\mathrm{n}=19)$, leiomyosarcoma $(\mathrm{n}=12)$, fibrosarcoma $(\mathrm{n}=8)$, rhabdomyosarcoma $(n=7)$, synvialsarcoma $(n=6)$, and others $(\mathrm{n}=8)$.

Concerning to the Enneking's classification [28], there were 30 (34.8\%) low-grade (stage I) and 52 (60.4\%) highgrade (stage II) tumours. In 4 cases distant metastases had to be observed (stage III).

The lower extremity was involved in 44 cases, the upper extremity in 26 cases, and the trunk in 16 cases, respectively.

In $35(40.7 \%)$ of the patients the tumour was primarily resected under the suspicion of a benign soft tissue tumour, in 31 of these cases the tumour was localized extracompartimentary. The histopathological examination revealed a sarcoma with an intralesional resection. These patients were admitted for further surgery to achieve a wide resection. The median duration between previous intralesional surgery and admission was 5.2 weeks (range,
2 to 16 weeks). There were 19 stage-I tumours, and 16 stage-II tumours in this group.

In 20 out of these patients we did not found any tumour tissue after the required secondary surgical procedure to achieve a wide margin. Thus, tumour samples were available only in 66 cases for microsatellite analysis.

All patients were informed that their data would be submitted for publication, and gave their consent.

\subsection{Oncologic Follow-Up}

All patients were routinely controlled clinically every 6 months up to five years postoperatively. Afterwards the patients were controlled yearly. The survival rate and the occurrence of local recurrence and metastases were determined. The survival probability was estimated by the method describe in [29]. The tumour stage, the tumour volume, the achieved surgical margin [28], and the fact of intralesional previous surgery were also analysed as prognostic factors.

\subsection{Microsatellite Analysis}

Tumour tissue samples were available in 66 cases. Specimes of formalin-fixed, paraffin embedded $7 \mu \mathrm{m}$ tissue sections containing both, tumour and surrounding normal tissue, were deparaffinized and stained with haemalaun. DNA was extracted using a microdissection device as previously described in [9]. The precipitated DNA was used for polymerase chain reactin (PCR) analysis.

\subsubsection{Analysis of Respective Microsatellites}

The technique commonly used at the former times of the study period is extensively described in [9].

PCR amplification was done on the following panel on 6 highly polymorphic microsatellite markers. The chosen oligonucleotide repeat markers should located in the neighborhood of well-known tumour suppressor genes ( $p 53$, $\mathrm{p} 16, \mathrm{rb} 1, \mathrm{hTR}$ ), or of the mismatch repair genes (hMSH2, hMLH1), respectively (genes linked with each marker are provided in parentheses):

D2S136 (hMSH2), D3S1076 (hMLH1), D17S250 (p53), D9S942 (p16), D13S153 (rb1), and D3S1246 (hTR) (9). PCR was performed in the Qiagen Hotstar Taq Mastermix kit (Fa. Qiagen, Hilden, Germany), and then products were separated using the ExcelGel ${ }^{\mathrm{TM}}$ DNA analysis kit (Fa. Amersham Biosciences, Munich, Germany). Products were silver-stained following the procedure outlined in [30].

A locus was considered informative for the respective polymorphism when normal tissue DNA exhibited two different alleles (heterozygosity). The intensities of the two alleles in the corresponding tumour were compared. The complete absence of an allele was interpreted as loss of heterozygosity $(\mathrm{LOH})$. Reduced intensity of one of the two alleles was considered to indicate allelic imbalance (AI). Appearence of new alleles in tumour samples was considered to indicate microsatellite instability (MSI). Two investigators evaluated the results of electrophoresis independently without any patient information. 


\subsection{Statistical Analysis}

Analysis of variance (ANOVA) was used to determine the oncologic outcome regarding the variables tumour volume, tumour stage, surgical margin, and the fact of intralesional previous surgery.

The correlation of the results of the detected genomic instabilities and the oncologic outcome was also analysed. Wilcoxon-test was used to compare subgroups. The survival probability was calculated by the method described in [29].

Statistical analysis of the data obtained was performed using the software SPSS 9.0 (SPSS Inc., Chicago, Il, USA). P-values $<0.05$ were considered significant.

\section{Results}

\subsection{Oncologic Results}

In the 30 low-grade tumours, the surgical margins achieved were wide in 21 cases, and marginal in 9 cases. In the 52 high-grade tumours the margins were radical in 7 , wide in 21 , marginal in 19, and intralesional in 5 cases. In the 4 stage-III tumours the margins were marginal in two cases, wide in one, and intralesional in one case. Amputations had to be performed in 9 patients (10.4\%): hemipelvectomy, the lower extremity, and the upper extremity, each in three times, respectively.

The median tumour volume of the 66 tumour containing specimens was $451 \mathrm{ml}$ (range, 3-4800 ml). The stage-I tumours were smaller $(386 \mathrm{ml})$ than the stage-II tumours $(442 \mathrm{ml})$, but the difference was not significant.

At the date of actual evaluation in January 2010, twentynine patients died of their disease, and 7 patients died of other causes.

The mean follow-up was 12 years (range, 10 - 16 years) or 144 months (range, 120 - 192 months), respectively. The median survival time was 58 months (range, 26-74 months). The patients presenting with stage III tumours had a median survival time of 15.25 months. Local recurrences were observed in 13 patients $(15.1 \%)$ after an average of 14.3 months (range, 4-47 months). Distant metastases were seen in 23 patients $(26.7 \%$ ) after an average of 11.7 months (range, 3-21 months). In most cases $(\mathrm{n}=21)$ there were pulmonary metastases.

Table 1 gives a survey of the oncological results in relation to the tumour stage and the surgical margin achieved.

The incidence of a local recurrence significantly depended upon the achieved surgical margin. The tumour volume had no influence.

Concerning all tumour stages, local recurrences were significantly more frequent in cases of achieved marginal margin compared with those of wide or radical margin. These findings could be stated in both subgroups, (i.e. in the low-grade as well as in the high-grade tumours). The incidence of distant metastases was higher in high-grade tumours than in low-grade-tumours. But in both tumour subgroups the incidence of distant metastases did not depend upon the surgical margin.

Table 1. Oncologic results in relation to the tumour stage and the surgical margin

\begin{tabular}{|c|c|c|c|c|c|c|c|c|c|c|}
\hline \multicolumn{3}{|c|}{ tumour stage } & \multicolumn{4}{|c|}{ surgical margin } & \multirow{2}{*}{ LR } & \multirow{2}{*}{ Met's } & \multirow{2}{*}{ DOD } & \multirow{2}{*}{ DOC } \\
\hline $\mathbf{I}$ & II & III & $\mathbf{r}$ & $\mathbf{w}$ & $\mathbf{m}$ & i & & & & \\
\hline \multirow[t]{7}{*}{30} & & & & 21 & & & & 1 & 1 & 1 \\
\hline & & & & & 9 & & 4 & 2 & 2 & 1 \\
\hline & 52 & & 7 & & & & 1 & 4 & 3 & \\
\hline & & & & 21 & & & 1 & 6 & 7 & 2 \\
\hline & & & & & 19 & & 6 & 6 & 9 & 2 \\
\hline & & & & & & 5 & 1 & & 3 & 1 \\
\hline & & 4 & & 1 & 2 & 1 & & 4 & 4 & \\
\hline 30 & 52 & 4 & 7 & 43 & 30 & 6 & 13 & 23 & 29 & 7 \\
\hline
\end{tabular}

(Abbreviations: r: radical; w: wide; m: marginal; i: intralesional; LR: local recurrence; Met`s: metastases; DOD: died of disease; DOC: died of other causes)

Excluding the four patients presenting with stage-III tumours, two different groups were also analyzed, the one $(n=35)$ with previous intralesional surgery, and the other $(n=47)$ without previous intralesional surgery. We found even less local recurrences and less distant metastases in the patients with previous intralesional surgery compared with those patients without previous surgery, but the difference was not significant. However, the achieved surgical margin was valued as wide in 20 cases of the 35 patients with previous intralesional surgery, where no tumour could be found at the histological examination.

In the 15 cases presented primarily with a local recurrence, there were no worse oncological results; in the most cases $(n=11)$ there were high-grade tumours. The local recurrences could be cured in 10 out of these 15 patients.

Thirteen patients developed local recurrences, in 10 of these cases within the first two post-operative years. The local recurrences could be cured in four cases, twice by amputation, and twice by a renewed resection.

Distant metastases developed in 23 patients; 6 out of them were treated surgically. Only two patients could be cured, one by resection of a peripheral soft tissue metastasis, the other is now for 148 months considered a complete responder after chemotherapy. All other patients with distant metastases died of their disease.

The Kaplan-Meier survival analyses in relation to the tumour stage and the achieved surgical margin are shown in table 2 and in table 3. 
Table 2. 5-year and 10-year survival probability in relation to the tumour stage

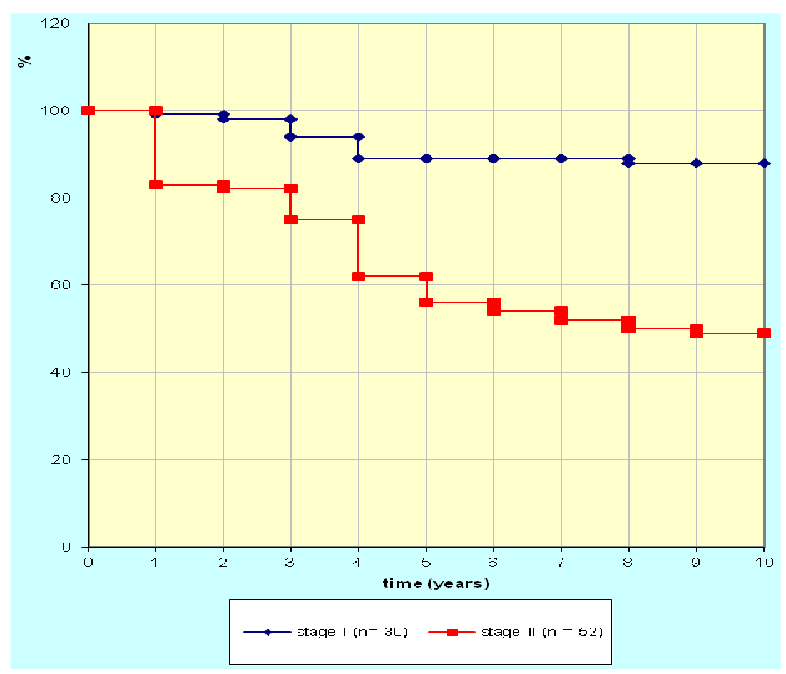

(Stage III tumours were excluded)

Table 3. 5-year and 10-year survival probability in relation to the surgical margin

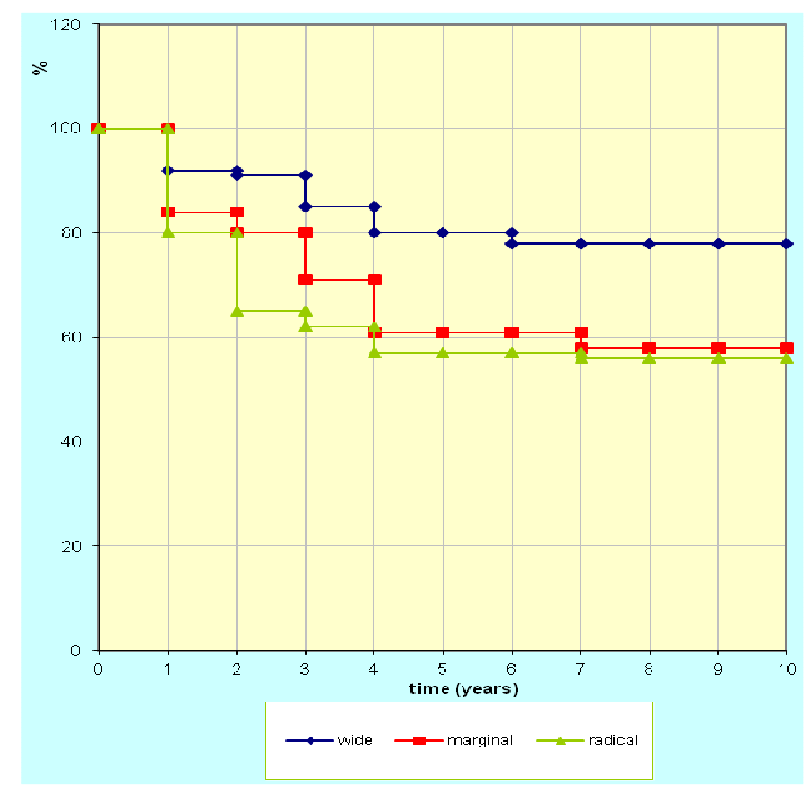

Excluding the stage-III tumours and the intralesional resected tumours, the overall survival was significantly dependent upon the tumour stage after 5 years as well as after 10 years. The survival probability of $56.5 \%$ after 5 years decreased in patients with high-grade sarcomas to $49 \%$ after 10 years, whereas the reduction of the survival probability during this period was insignificant in patients with low-grade sarcomas $(89.5 \%$ to $88 \%)$.

Patients underwent wide resection showed a better prognosis than patients with marginal resection, but the difference was not significant. In both groups, there was a similar reduction of the survival probability in the period of 5 years to 10 years postoperatively. The worse prognosis after radical resections can be explained by the fact that there were only high-grade tumours in this group.

There were also no differences in the 5-year and the 10year survival rate concerning the tumour volume, the fact of previous intralesional surgery, the primary presentation with a local recurrence, or the fact of chemotherapy in high-grade sarcomas.

\subsection{Molecular Biological Results}

In 66 patients tumour tissue was available for microsatellite analysis. Thus, a total of 396 loci were investigated, 130 of them $(32.8 \%)$ were non-informative. In the remaining 266 loci, 94 of them $(35.4 \%)$ were without pathological findings, i.e. negative.

Overall, allelic changes could be found in 172 out of the informative loci $(64.6 \%)$, in $79.6 \%$ of the high-grade sarcomas, and in $13.3 \%$ of the low-grade-sarcomas.

MSI was detected only in 18 out of the 266 informative loci (6.8\%); all of them were in high-grade sarcomas. LOH was seen in 50 loci $(18.8 \%)$, in one out of the 60 informative loci $(1.7 \%)$ of the low-grade sarcoams, whereas in 49 loci out of the 206 informative loci $(23.8 \%)$ of the high-grade sarcoams. This difference was statistically significant. Allelic imbalances (AI) were found in 39\% out of all loci, in $11,6 \%$ of the low-grade sarcomas, and in $47,1 \%$ of the high-grade sarcomas, respectively.

Table 4 summarizes the results of the genomic instability regarding the tumour stage.

(Stage III tumours and intralesional resections were excluded)

Table 4. Microsatellite analysis in relation to the tumour stage

\begin{tabular}{|c|c|c|c|c|}
\hline & & total & low-grade (stage I) & high-grade(stage II) \\
\hline \multirow{9}{*}{ loci } & $\mathrm{n}$ & 66 & 15 & 51 \\
\hline & total & 396 & 90 & 306 \\
\hline & informative & 266 & 60 & 206 \\
\hline & negative & $94(35,4 \%)$ & $52(86,7 \%)$ & $42(20,4 \%)$ \\
\hline & genomic & & & \\
\hline & instabilities & $172(64,6 \%)$ & $8(13,3 \%)$ & $164(79,6 \%)$ \\
\hline & MSI & $18 \quad(6,4 \%)$ & - & $18 \quad(8,7 \%)$ \\
\hline & $\mathrm{LOH}$ & $50(18,8 \%)$ & $1(1,7 \%)^{*}$ & $49(23,8 \%)^{*}$ \\
\hline & AI & $104(39 \%)$ & $7(11,6 \%)$ & $97(47,1 \%)$ \\
\hline
\end{tabular}

(Abbreviations: MSI: microsatellite instability; LOH: loss of heterozygosity; AI: allelic imbalance; *: statistically significant (p<0.05)) 
Table 5. Survival rate and genetic instability categorized by different types of STS

\begin{tabular}{|c|c|c|c|c|c|c|c|c|}
\hline \multirow[t]{2}{*}{ histology } & \multirow[t]{2}{*}{ n } & \multirow[t]{2}{*}{$\begin{array}{l}\text { 5-year } \\
\text { survival }\end{array}$} & \multirow[t]{2}{*}{$\begin{array}{l}\text { 10-year } \\
\text { survival }\end{array}$} & \multirow[t]{2}{*}{$\begin{array}{l}\text { specimen } \\
\text { available for } \\
\text { MSI analysis }\end{array}$} & \multirow[t]{2}{*}{ informative loci } & \multicolumn{3}{|c|}{$\begin{array}{l}\text { thereof } \\
\text { genetic instabilities }\end{array}$} \\
\hline & & & & & & MSI & $\mathrm{LOH}$ & $\mathrm{AI}$ \\
\hline MFH & 25 & $70.5 \%$ & $66 \%$ & 20 & 80 & 5 & 15 & 32 \\
\hline liposarcoma & 18 & $67.5 \%$ & $62 \%$ & 14 & 61 & 4 & 13 & 27 \\
\hline leiomyosarcoma & 11 & $64 \%$ & $60.5 \%$ & 9 & 44 & 2 & 5 & 9 \\
\hline fibrosarcoma & 7 & $68 \%$ & $63 \%$ & 6 & 28 & 2 & 6 & 10 \\
\hline rhabdomyosarcoma & 7 & $68.5 \%$ & $61 \%$ & 6 & 22 & 2 & 7 & 15 \\
\hline others & 14 & $70.4 \%$ & $62 \%$ & 11 & 31 & 3 & 7 & 11 \\
\hline total & 82 & $\varnothing 68.5 \%$ & $\varnothing 63.2 \%$ & total 66 & 266 & 18 & 50 & 104 \\
\hline
\end{tabular}

(Abbreviations: MFH: malignant fibrous histiocytoma; MSI: microsatellite instability; LOH: loss of heterozygosity; AI: Allelic imbalance; stage III tumours were excluded)

Correlation of the presence of genomic instability and the patient's age, the patient's sex, the tumour volume, the localization of the tumour, or the histologic type of the tumour were not detected.

The number of each tumour type was too small to allow an inference about the survival analysis as well as about the role of genetic instabilities. Die descriptive results of the different tumour types concerning the survival rate and the molecular biological findings are shown in table 5 .

Concerning the different markers, the highest rate of genomic instability was seen at the microsatellite nearby hMLH1 (78\%), hTR (75.5\%), and hMSH2 (70.2\%). The lowest validity was seen at the marker nearby p16 (40\%).

It was particulary noticeably and statistically significant, that 15 out of the 18 cases of MSI were localized at the marker in the neighborhood of the mismatch repair genes, i.e. hMSH2 and hMLH1. The frequency of different genomic instabilities in relation of the different markers is demonstrated in table 6 .

Table 6. Frequency of different genomic instabilities in relation to the individual markers

\begin{tabular}{llllll}
\hline \multirow{2}{*}{ markers } & \multirow{2}{*}{ (linked genes) } & \multicolumn{5}{c}{ genomic instabilities } \\
\cline { 3 - 6 } & & total & MSI & LOH & AI \\
\hline D2S136 & (hMSH2) & 26 & $12^{*}$ & 11 & 3 \\
D3S1076 & (hMLH1) & 32 & 3 & 12 & 17 \\
D17S250 & $(\mathrm{p} 53)$ & 34 & 3 & 13 & 18 \\
D9S942 & $(\mathrm{p} 16)$ & 18 & & & 18 \\
D13S153 & $(\mathrm{rb} 1)$ & 28 & & 3 & 25 \\
D3S1246 & $(\mathrm{hTR})$ & 34 & & 11 & 23 \\
total & & 172 & 18 & 50 & 104 \\
\hline
\end{tabular}

(Abbreviations: MSI: microsatellite instability; LOH: loss of

heterozygosity; AI: allelic imbalance; *: statistically significant $(\mathrm{p}<0.05))$

\subsection{Correlation of Oncologic and Molecular Biological Results}

As expected, the low-grade sarcomas presented significantly better oncologic outcome. There was a low rate of genomic instability in these cases, only 8 out of the 60 informative loci (13.3\%) were positive. Excluding the stage III sarcomas, the remaining 47 high-grade sarcomas presented genomic instabilities in $79.6 \%$ of the informative loci. Only in one of these cases a solitary alteration could be found showing $\mathrm{LOH}$ at the marker nearby $\mathrm{hMSH} 2$. In all other cases there were multiple genetic alterations at different corresponding markers, in 7 cases at 2 markers, in 15 at 3 , in 20 at 4, and in two cases at 5 markers, respectively.

The low frequency of MSI in high-grade sarcomas (8.7\%) had no influence on the overall survival. The appeareance of allelic imbalance was also considered to be non-specific.

But there was a significant influence of the LOH concerning the survival probability in the high-grade sarcomas. When $\mathrm{LOH}$ was detected at least at one marker, the 5-year and 10-year survival probabilities were $48.6 \%$, in cases without $\mathrm{LOH}$ detectable.

There was no significant difference concenring the detection of LOH at the different markers or the frequency of LOH at several markers. Excluding the markers nearby p16 and rb1, there was no preference of any marker, where $\mathrm{LOH}$ could be detected.

\section{Discussion}

Soft tissue sarcomas are very rare tumours accounting for less than $1 \%$ of all malignancies in the adult [30, 31, 32]. Basically, they can be divided into low-grade and highgrade tumours by the means of histopathological criteria resulting in the surgical classification according to [28].

In all malignancies, every treatment modality has to be measured by the achieved oncologic outcome. In sarcoams, surgery is considered to play the most significant role. Different prognostic factors are reported in STS $[31,32]$.

The overall-survival mostly depends upon the tumour stage. The 5-year-survival probabilities range from 50-60\% in stage-II tumours up to $80-90 \%$ in stage-I tumours [31, 32]. These reports are confirmed by our findings, $89.5 \%$ in stage-I tumours, and $56.5 \%$ in stage-II tumours. Most studies reported an oncologic follow-up of 5 years [31 - 34]. But we could demonstrate in our series, that the survival probability will decrease furthermore. Low-grade sarcomas showed an insignificant decrease of survival probability from $89.5 \%$ after 5 years to $88 \%$ after 10 years, but highgrade sarcomas from $56.5 \%$ after 5 years to $49 \%$ after 10 years, respectively

There are some other prognostic factors in soft tissue sarcomas reported in the literature, such as tumour type, tumour volume, and localization [31,32]. We could not 
confirm these observations, maybe due to the limited number of patients in our series. The overall survival in our series only depended upon the tumour stage. The low-grade sarcomas were on average smaller than the high-grade sarcomas (386 $\mathrm{ml}$ versus $442 \mathrm{ml}$ ), but the difference was not significant. The thought can be suggested, that lowgrade sarcomas were smaller in our series and therefore resectable more frequently with a wide margin. However, we could not find a statistical significance. The 5-year survival rate was $60.7 \%$, when a marginal margin was achieved, and it was $80 \%$, when a wide margin was feasible, the 10 -year survival rate was $58 \%$ and $78 \%$, respectively.

But we agree with other authors [31, 35], that the achieved surgical margin is the most important prognostic factor for local tumour control. The incidence of a local recurrence was significantly higher in tumours resected with a marginal margin compared with those resected with a wide margin. We observed significantly more local recurrences, when only a marginal margin was feasible.

Some studies $[31,34]$ demonstrated that the occurrence of local recurrence promotes the development of distant metastases resulting in a poor overall survival. In our series, we saw 15 patients presented with a local recurrence without concomitant distant metastases. Ten of them could be cured, and we could not find a worse oncologic outcome. But the small number of cases may not allow any statistical predication.

Despite intensive search of clinical and histomorphologic criteria individual prognostic statements are often not feasible in STS due to their considerable variability. Therefore, further predictive markers are intensively searched to allow a more precise prediction of the prognosis, or to define patient groups, who may benefit from specific treatment modalities.

The role of genetic instability is well known in different cancers [2, 16 - 18].

The two phenomena, microsatellite instability (MSI) and loss of heterozygosity (LOH), are considered to be of great importance [1 - 3, 8, 10 - 13, 24].

MSI is considered as a dysfunction of the mismatch repair (MMR) genes [5, $10-12,14]$, whereas LOH seems to correspond to genetic defects of tumour suppressor genes $[7,16,21,22,24,26,27]$.

Thus, MSI is reported as a negative prognostic factor in different cancers, such as lung cancer, or colorectal cancer [16 - 18]. It is considered to be a phenomenon of the early phase of tumour progression [10]. LOH is also accepted to be a negative predictor in many cancers. The colorectal cancer is the most frequently investigated tumour [16, 17].

The role of MSI and LOH in STS is still unclear. The most molecular-biological and cytogenetic data are reported in ostesarcomas, in Ewing's sarcomas, or in uterine sarcomas [3, 4, 6, 24, 26, 27].

As described in [4], there was no MSI in 29 bone sarcomas at 6 different loci. MSI was also not found in 28 liposarcomas at 9 different loci, but LOH was present in 11 tumours (39\%) [7]. MSI was found in only 3 cases out of
39 STS examined for 12 different loci, whereas LOH for at least one marker was seen in $14 \%$ to $85 \%$ depending upon the tumour type [3].

On the other hand, another study [21] reported on 20 sarcomas demonstrating MSI in 5 out of 7 high-grade tumours using 8 different markers. The region of chromosome 12 was especially affected. The oncogene $\mathrm{mdm} 2$ is localized in the neighborhood of the described microsatellite, it is considered to be the main antagonist of the tumour suppressor gene p53. All low-grade sarcomas did not show any MSI. LOH was seen in $75 \%$ of the highgrade sarcomas at the chromosome 2 in the region of the locus nearby the hMSH2 gene.

The detection of LOH was described as a negative predictor in 31 patients with thoracic sarcomas [8].

As confirmed by the findings in our series presenting MSI in only $8 \%$ of the informative loci without any prognostic value, MSI seems to play no prominent role in STS.

But the rate of $\mathrm{LOH}$ in our series corresponds with the findings of other authors $[7,21,24,26]$. The incidence of $\mathrm{LOH}$ correlated to the tumour stage. There was no relationship to any histological subtype of STS, but a statistical valid statement is not feasible due to the small number of patient in each histological subtype. In our series, the oncologic outcome was worse in the high-grade sarcomas, when LOH was presented.

Many studies describe a correlation between MSI and alterations of the mismatch-repair-genes hMSH2 and hMLH1 [6, 11, 12, 15, 19]. As reported in [11], MSI at 5 different markers were seen in $25 \%$ of 40 STS. In $50 \%$ of these cases, loss of expression of the products of the hMLH1- and hMSH2-genes had to be observed. Another study [19] revealed similar results. MSI was visible in $37.5 \%$ of 16 alveolar sarcomas, whereas in two thirds of these cases loss of expression of the gene products of hMLH1 and hMSH2 were notified.

In our series, the important role of the mismatch repair genes (hMLH1 and hMSH2) was also confirmed showing 15 of the $18 \mathrm{MSI}$, and 23 of the $50 \mathrm{LOH}$, respectively, detected at the loci nearby these genes.

The role of the tumour suppressor gene p53 is also extensively described in STS. The frequency of mutation is reported to amount $15 \%$ to $20 \%$ [23]. In the balance of oncogenes and tumour suppressor genes, p53 is considered the antagonist of $\operatorname{mdm} 2[2,22,23,25]$. The high significance of the co-expression of $\mathrm{p} 53$ - and of mdm2gene products as an independent molecular prognostic factor is reported in [25]. Thus, the thesis is confirmed, that a medium transcription rate of oncogenes and tumour suppressor genes is wanted for a physiologic cellular regulation process. The imbalance of this transcription rate seems to have an oncogenic potential [25].

There exist only a few reports on genomic instabilities of the microsatellites located nearby the rb1- and the p16genes. In our series, we found no MSI for both markers, and only $10 \% \mathrm{LOH}$ at the locus nearby the rbl gene. As reported in [26] about 47 osteosarcomas, the 5-year 
survival was significantly reduced in cases of detected LOH nearby the rblgene locus. These findings were recently confirmed by another study [24]. Allelic loss at the p16 gene locus was detected in 5.1\% and MSI was reported in $25 \%$ of cases in the large series of 135 sarcomas [36]. These results are confirmed by the findings in our series. Alterations of the microsatellite nearby the p16 gene locus were observed in $40 \%$, but there were only non-specific allelic imbalances, LOH and MSI were not detected.

Thus, we assume that genetic alterations at the p16 gene locus seem to be not relevant to the oncogenesis or to the tumour progression in sarcomas.

The role of the hTR gene in STS is unclear. Related LOH was found in $50 \%$ of osteosarcomas [27] and in $20 \%$ of leiomyosarcomas [9]. In our series, we observed genomic alterations in $75 \%$ at the microsatellite located nearby the hTR gene, in one third of theses cases presented as MSI.

\section{Conclusions}

The surgical margin achieved after resection of a sarcoma is the most important factor for local tumour control, whereas the overall survival mainly depends upon the tumour stage. In high-grade sarcomas the survival rate will drop even after 5 years.

Therefore, long-term studies with prolonged follow-up will be helpful to define the exact oncologic outcome.

The role of the phenomena of the genetic alteration, microsatellite instability and loss of heterozygosity, are still unclear. MSI seems to be a rare phenomenon in STS without any prognostic value, but LOH indicates a worse prognosis in high-grade sarcomas.

The value of the different tumour suppressor genes has to be investigated by further multicentric studies. To assess the relevance of MSI and LOH for tumour progression and oncogenesis in sarcomas, larger numbers, modern detection techniques, and an appropriate microsatellite loci panel will be essential.

\section{References}

[1] Fletcher CD, Fletcher JA, Cin PD, Landanyi M, Woodruff JM. Diagnostic gold standard for soft tissue tumours: morphology or molecular genetics? Histopathology 2001; 39: $100-103$.

[2] Lengauer C, Kinzler KW, Vogelstein B. Genetic instabilities in human cancers. Nature 1998; 396: 643-649.

[3] Suwa K, Ohmori M, Miki H. Microsatellite alterations in various sarcomas in Japanese patients. J Orthop Sci 1999; 4: 223-230.

[4] Tarkannen M, Aaltonen LA, Böhling T, Kivioja A, Karaharju E, Elomaa I, et al. No evidence of microsatellite instability in bone tumours. Br J Cancer 1996; 74: 453-455.

[5] Martin SS, Hurt WG, Hedges LK, Butler MG, Schwartz HS. MIN in sarcomas. Ann Surg Oncol 1998; 5: 356-360.
[6] Alldinger I, Schaefer KL, Goedde D, Ottaviano L, Dirksen U, Ranft A, et al. Microsatellite instability in Ewing tumor is not associated with loss of mismatch repair protein expression. J Cancer Res Clin Oncol 2007; 133: 749-759.

[7] Schneider-Stock R, Szibor R, Walter H, Plate I, Roessner A. No microsatellite instability but frequent $\mathrm{LOH}$ in liposarcoma. Int J Oncol 1999; 14: 721-726.

[8] Iyoda A, Hiroshima K, Toyozaki T, Baba M, Fujisawa T, Yusa T, et al. Microsatellite alterations in patients with thoracic sarcomas. Oncol Rep 2001; 8: 917-921.

[9] Verelst S, Hans J, Hanselmann R, Wirbel RJ. Genetic instability in primary leiomyosarcoma of bone. Hum Pathol 2004; 35: 1404-1412.

[10] Maehara Y, Oda S, Sugimachi K. The instability within. Problems in current analyses of microsatellite instability. Mutat Res 2001; 461: 249-263.

[11] Kawaguchi K, Oda Y, Takahira T, Saito T, Yamamoto H, Kobayashi C, et al. Microsatellite instability and hMLH1 and hMSH2 expression analysis in soft tissue sarcomas. Oncol Rep 2005; 13: 241-246.

[12] Sin KH, Park JG. Microsatellite instability is associated with genetic alterations but not with low levels of expression of the human mismatch repair proteins hMSH2 and hMLH1. Eur J Cancer 2000; 36: 925-931.

[13] Stahl J. Mismatch repair proteins and microsatellites hit clinical practice. Adv Ant Pathol 2000; 7: 85-93.

[14] Garcia JJ, Kramer MJ, O`Donnell RJ, Horvai AE. Mismatch repair protein expression and microsatellite instability: a comparison of clear cell sarcoma of soft parts and metastatic melanoma. Modern Pathology 2006; 19: 950-957.

[15] Ericson K, Engellau J, Petersson A, Lindblom A, Domanski $\mathrm{H}$, Akerman $\mathrm{M}$, et al. Immunhistochemical loss of DNA mismatch repair proteins $\mathrm{MSH} 2$ and MSH6 in malignant fibrous histiocytomas. Sarcoma 2004; 8: 123-127.

[16] Chang SC, Lin JK, Lin TC, Liang WY. Loss of heterozygosity: an independent prognostic factor in colorectal cancer. Word J Gastroenterol 2005; 14: 778-784.

[17] Choi SW, Lee KL, Bae YA, Min KO, Kwon MS, Kim KM, et al. Genetic classification of colorectal cancer based on chromosomal loss and microsatellite instability predicts survival. Clin Cancer Res 2002; 8: 2311-2322.

[18] Adachi JI, Shiseki M, Okazaki T, Ishimaru G, Noguchi M, Hirohani S, et al. Microsatellite instability in primary and metastatic lung carcinomas. Genes Chromosomes Cancer 1996; 14: 301-306.

[19] Saito T, Oda Y, Kawaguchi K, Takahira T, Yamamoto H, Sakamoto A, et al. Possible association between tumorsuppressor gene mutations and hMSH2/hMLH1 inactivation in alveolar soft part sarcoma. Hum Pathol 2003; 34: 841-849.

[20] Sabah M, Cummins R, Leader M, Kay E: Leiomyosarcoma and malignant fibrous histiocytoma share similar allelic imbalance pattern at 9p. Virch Arch 2005; 446: 251-258.

[21] Rucinska M, Kozlowski L, Pepinski W, Shawronska M, Janinca J, Wojtukiewicz MZ. High grade sarcomas are associated with microsatellite instability (chromosome 12) and loss of heterozygosity (chromosome 2). Med Sci Monit 2005; 11: 65-68. 
[22] Taubert H, Schuster K, Brinck U, Bartel F, Kappler W, Lautenschlager C, et al. Loss of heterozygosity at 12Q14-15 often occurs in stage I soft tissue sarcomas and is associated with MDM2 amplification in tumors at various stages. Mod Pathol 2003; 16: 1109-1116.

[23] Yoo J, Lee HK, Kang CS, Park WS, Lee JY, Shim SI. P53 gene mutation and p 53 protein expression in human soft tissue sarcomas. Arch Pathol Lab Med 1997; 121: 395-399.

[24] Heinsohn S, Evermann U, Zur Stadt U, Bielack S, Kabisch $\mathrm{H}$. Determination of the prognostic value of loss of hetreozygosity at the Retinoblastoma gene in osteosarcoma. Int J Oncol 2007; 30: 1205-1214.

[25] Würl P, Taubert H, Bache M, Koll J, Meye A, Berger D, et al. Frequent occurrence of p53 mutations in rhabdomyosarcoma and leiomyosarcoma, but not in fibrosarcoma and malignant neural tumors. Int $\mathrm{J}$ Cancer 1996; 69: 317-323.

[26] Fuegas O, Guriec N, Babin-Boilletot A, Marcellin L, Simon $\mathrm{P}$, Babin S. Et al. Loss of heterozygosity of the RB gene is a poor prognostic factor in patients with osteosarcoma. J Clin Oncol 1996; 14: 467-472.

[27] Patino-Garcia A, Pineito ES, Diez MZ, Iturriagagoitia LG, Klussmann FA, Ariznabarreta LS. Genetic and epigenetic alterations of the cell cycle regulators and tumor suppressor genes in pediatric osteosarcomas. J Pediatr Hematol Oncol 2003; 25: 362-367.

[28] Enneking W. A system of staging musculoskeletal neoplasms. Clin Orthop 1986; 204: 9-24.
[29] Kaplan E, Meier P. Nonparametric estimation for incomplete observation. Am J Stat Assoc 1952; 52: 457-481.

[30] Bassam B, Caetano-Anolles G, Gresshoff PM. Fast and sensitive silver staining of DNA in polyacylamide gels. Anal Biochem 1991; 16: 80-83.

[31] Pisters PW, Leung DH, Woodruff JM, Shi W, Brennan MF. Analysis of prognostic factors in 1041 patients with localized soft tissue sarcoma on the extremity. J Clin Oncol 1996; 14: 1679-1689.

[32] Chang HR, Gaynor J, Tan C, Hajdu SI, Brennan MF. Multifactorial analysis of survival in primary extremity liposarcoma. Word J Surg 1990; 14: 610-618.

[33] Davidge K, Bell R, Ferguson P, Turcotte R, Wunder J, Davis AM. Patient expectations for surgical outcome in extremity soft tissue sarcoma. J Surg Oncol 2009; 100: 375-381.

[34] Aksnes LH, Bauer HC, Jebsen NL, Folleras G, Allert C, Haugen GS, et al. Limb-sparing surgery preserves more function than amputation: a Scandinavian sarcoma group study of 118 patients. J Bone J Surg 2008; 90B: 786-794.

[35] Lewis JJ, Leung D, Espat J, Woddruff JM, Brennan MF. Effect of resection in extremity soft tissue sarcoma. Ann Surg 2000; 231: 655-663.

[36] Schneider-Stock R, Walter H, Haeckel C, Radig K, Rys J, Roessner A. Gene alterations at the CDKN2A (p16/MTS1) locus in soft tissue tumours. Int J Oncol 1998; 13: 325-329. 